\title{
The Problem of a Self-Gravitating Scalar Field
}

\author{
Demetrios Christodoulou ${ }^{\star}$ \\ Department of Mathematics and Physics, Syracuse University, Syracuse, New York USA
}

\begin{abstract}
In this paper we begin the study of the global initial value problem for Einstein's equations in the spherically symmetric case with a massless scalar field as the material model. We reduce the problem to a single nonlinear evolution equation. Taking as initial hypersurface a future light cone with vertex at the center of symmetry, we prove, the local, in retarded time, existence and global uniqueness of classical solutions. We also prove that if the initial data is sufficiently small there exists a global classical solution which disperses in the infinite future.
\end{abstract}

\section{Section 0. Introduction}

The global initial value problem is the fundamental problem of any classical physical theory. In general relativity this problem has not been solved even in the small, as the stability of Minkowski spacetime is still an open question. This is the question of whether initial data for the Einstein equations, which is in some sense sufficiently close to the trivial data, gives rise to a geodesically complete spacetime which is asymptotic to the Minkowski spacetime in the infinite future. The PenroseHawking singularity theorems show that if a spacetime contains a closed trapped surface, then it cannot be null or timelike geodesically complete [1-3]. Perhaps the most fundamental open problem in general relativity is the so-called cosmic censorship question, which may be formulated as follows: whether, given arbitrary asymptotically flat initial data, there exists a solution of the Einstein equations which is a globally hyperbolic spacetime posessing a complete future null infinity. This question is part of the global initial value problem in the large. A further part, of great interest, is the study of the formation of event horizons.

We believe the study of these problems under the simplifying assumptions of spherical or axial symmetry is important in order to develop methods which may ultimately lead to the resolution of the general problems. With this motivation, we

* Research supported in part by National Science Foundation grants MCS-8201599 to the Courant Institute and PHY-8318350 to Syracuse University 
begin here the study of the Einstein equations in the spherically symmetric case with a massless scalar field as our material model. The material equation in a given spacetime is then the linear wave equation in that spacetime. The problem is however nonlinear in an essential way, as the spacetime. metric is itself determined through the Einstein equations by the scalar field. Many of the difficulties involved in solving the basic problems which we have stated are already present in our model. Among spherically symmetric models, this model is most analogous to the axially symmetric problem for the vacuum Einstein equations. The role of the scalar field is played there by the dynamical degrees of freedom of the spacetime itself, namely the gravitational radiation field.

The plan of the present paper is the following: In Sect. 1 we introduce in a geometrical way a coordinate system which exactly covers the domain of outer communications. In Sect. 2 we give the components of the curvature tensor, the Ricci tensor and the energy-momentum tensor, and using the Bianchi identities we reduce Einstein's equations to three equations, two for the two metric functions and one for the scalar field. In Sect. 3 we eliminate the metric functions in terms of the scalar field and we reduce the whole problem to a single nonlinear evolution equation. In Sect. 4 we define the local mass and give its evolution law. In Sect. 5 we derive the properties of the incoming light rays which are the characteristics of the problem. In Sect. 6 we consider the limiting hypersurface $u=\infty$, where $u$ is the retarded time, and we show that the part of this hypersurface exterior to a certain sphere represents future timelike infinity. In Sect. 7 we set up the initial value problem for the nonlinear evolution equation taking as initial hypersurface a future light cone with vertex at the center of symmetry. We then prove the local, in retarded time, existence of classical solutions to the problem. In Sect. 8 we prove the global uniqueness of classical solutions. Finally, in Sect. 9 we prove the main result of this paper (Theorem 3 ), the global existence of classical solutions for sufficiently small initial data. These solutions decay in a certain way as $u \rightarrow \infty$, and the corresponding spacetimes are timelike and null geodesically complete and are asymptotic to the Minkowski spacetime in the infinite future. The proof of this result is based on the use of the Banach space $X^{\prime}$ defined there.

In a subsequent paper we shall study the global problem for arbitrarily large initial data.

\section{Section 1. The Coordinate System}

We consider a spacetime, which is the manifold $\mathbb{R}^{4}$ together with a Lorentz metric, which is time and space oriented and on which the rotation group $\mathrm{SO}(3)$ acts as an isometry group. We assume that there is a central (timelike) world line which is invariant by the group, and that the group orbits through any point not on the central world line are spacelike 2-spheres. These 2-spheres are then metric 2-spheres. Through each point on a given 2-sphere there passes a timelike half-plane, the boundary of which is the central world line, which is orthogonal to the group orbits and intersects each group orbit at a single point. The rotation group maps these halfplanes isometrically onto each other. The quotient of the spacetime by the group is the Lorentzian 2-manifold with boundary represented by any one of the half-planes. 
Let $A$ denote the area of the group orbits. We assume that the future directed null geodesics through any point on the central world time are complete, and that the area $A$ of the group orbit through each point on such a null geodesic is a monotonically increasing function of the affine parameter, tending to infinity as the affine parameter tends to infinity. We define then a coordinate $r$ by setting $A=4 \pi r^{2}$. (The central world line is the set $r=0$.) We assume that the world lines $r=r_{0}$ (constant) in each half-plane are all timelike. We define then a coordinate $u$ by requiring that $u$ is constant on the future light cone of each point on the central world line, and that on a world line $r=r_{0}, u$ tends to the proper time as $r_{0} \rightarrow \infty$. With the above assumptions, the metric of the spacetime can be expressed in the form:

$$
d s^{2}=-e^{2 v} d u^{2}-2 e^{v+\lambda} d u d r+r^{2} d \Sigma^{2},
$$

where the first part is the metric on the quotient and $d \Sigma^{2}$ is the canonical metric of $S^{2}$. Here $v$ and $\lambda$ are functions of $u$ and $r$ and $v \rightarrow 0$ for $r \rightarrow \infty$ at each $u$. As we shall consider only spacetimes which are asymptotically flat at future null infinity, we require also $\lambda \rightarrow 0$ for $r \rightarrow \infty$ at each $u$. As we are interested only in the future of some initial future light cone with vertex at the center, the coordinates $u$ and $r$ shall range in the quadrant $0 \leqq u<\infty, 0 \leqq r<\infty$, where $u=0$ corresponds to the initial future light cone.

The coordinate system we have constructed is known as a Bondi coordinate system [4]. The spacetime we are considering will in general be extendible to a larger spacetime of which it will be the domain of outer communications (see Sect. 6).

We introduce now the null tetrad $\left(n, l, \zeta_{1}, \zeta_{2}\right)$, where

$$
n=e^{-v} \frac{\partial}{\partial u}-\frac{1}{2} e^{-\lambda} \frac{\partial}{\partial r}, \quad l=e^{-\lambda} \frac{\partial}{\partial r}
$$

and $\left(\zeta_{1}, \zeta_{2}\right)$ is a (locally defined) orthonormal frame on $S^{2}$. The vector fields $n$ and $l$ are null, the integral curves of $n$ are the incoming light rays and the integral curves of $l$ are the outgoing light rays. Both $n$ and $l$ are orthogonal to $\zeta_{1}$ and $\zeta_{2}$ and $g(n, l)=$ -1 . The inverse metric can then be expressed in the form:

$$
g^{\mu v}=-n^{\mu} l^{v}-l^{\mu} n^{v}+\zeta_{1}^{\mu} \zeta_{1}^{v}+\zeta_{2}^{\mu} \zeta_{2}^{v} .
$$

\section{Section 2. The Einstein Equations}

The only nonvanishing components of the Riemann curvature tensor of the metric (1.1) with respect to the null tetrad $\left(n, l, \zeta_{1}, \zeta_{2}\right)$ are: 1$)$ The sectional curvature $K(n, l)$ of the $(n, l)$ plane:

$$
\begin{aligned}
K(n, l):= & R(n, l, n, l)=-e^{-v-\lambda}\left(\frac{\partial^{2} v}{\partial u \partial r}+\frac{\partial^{2} \lambda}{\partial u \partial r}\right) \\
& +e^{-2 \lambda}\left[\frac{\partial^{2} v}{\partial r^{2}}+\left(\frac{\partial v}{\partial r}-\frac{\partial \lambda}{\partial r}\right) \frac{\partial v}{\partial r}\right] .
\end{aligned}
$$

2) The sectional curvatures $K\left(n, \zeta_{1}\right):=R\left(n, \zeta_{1}, n, \zeta_{1}\right)$ and $K\left(n, \zeta_{2}\right):=R\left(n, \zeta_{2}, n, \zeta_{2}\right)$ of 
the $\left(n, \zeta_{1}\right)$ and $\left(n, \zeta_{2}\right)$ planes:

$$
K\left(n, \zeta_{1}\right)=K\left(n, \zeta_{2}\right)=\frac{1}{r}\left[-e^{-v-\lambda} \frac{\partial \lambda}{\partial u}+\frac{1}{4} e^{-2 \lambda}\left(\frac{\partial v}{\partial r}+\frac{\partial \lambda}{\partial r}\right)\right] .
$$

3) The sectional curvatures $K\left(l, \zeta_{1}\right):=R\left(l, \zeta_{1}, l, \zeta_{1}\right)$ and $K\left(l, \zeta_{2}\right):=R\left(l, \zeta_{2}, l, \zeta_{2}\right)$ of the $\left(l, \zeta_{1}\right)$ and $\left(l, \zeta_{2}\right)$ planes:

$$
K\left(l, \zeta_{1}\right)=K\left(l, \zeta_{2}\right)=\frac{e^{-2 \lambda}}{r}\left(\frac{\partial v}{\partial r}+\frac{\partial \lambda}{\partial r}\right)
$$

4) The sectional curvature $K\left(\zeta_{1}, \zeta_{2}\right):=R\left(\zeta_{1}, \zeta_{2}, \zeta_{1}, \zeta_{2}\right)$ of the $\left(\zeta_{1}, \zeta_{2}\right)$ plane:

$$
K\left(\zeta_{1}, \zeta_{2}\right)=\frac{1}{r^{2}}\left(1-e^{-2 \lambda}\right)
$$

And finally,

5) The components

$$
R\left(n, \zeta_{1}, l, \zeta_{1}\right)=R\left(n, \zeta_{2}, l, \zeta_{2}\right)=\frac{e^{-2 \lambda}}{2 r}\left(\frac{\partial v}{\partial r}-\frac{\partial \lambda}{\partial r}\right) .
$$

The only nonvanishing components of the Ricci tensor are then given by:

$$
\begin{aligned}
& R(n, n)=K\left(n, \zeta_{1}\right)+K\left(n, \zeta_{2}\right), \\
& R(n, l)=K(n, l)+R\left(n, \zeta_{1}, l, \zeta_{1}\right)+R\left(n, \zeta_{2}, l, \zeta_{2}\right), \\
& R(l, l)=K\left(l, \zeta_{1}\right)+K\left(l, \zeta_{2}\right), \\
& R\left(\zeta_{1}, \zeta_{1}\right)=-2 R\left(n, \zeta_{1}, l, \zeta_{1}\right)+K\left(\zeta_{1}, \zeta_{2}\right), \\
& R\left(\zeta_{2}, \zeta_{2}\right)=-2 R\left(n, \zeta_{2}, l, \zeta_{2}\right)+K\left(\zeta_{1}, \zeta_{2}\right),
\end{aligned}
$$

and the scalar curvature is:

$$
R=-2 R(n, l)+R\left(\zeta_{1}, \zeta_{1}\right)+R\left(\zeta_{2}, \zeta_{2}\right)
$$

Thus, the only nonvanishing components of the Einstein tensor $G_{\mu v}=R_{\mu \nu}-\frac{1}{2} g_{\mu \nu} R$ are:

$$
\begin{aligned}
G(n, n)=R(n, n) & =\frac{1}{r}\left[-2 e^{-v-\lambda} \frac{\partial \lambda}{\partial u}+\frac{1}{2} e^{-2 \lambda}\left(\frac{\partial v}{\partial r}+\frac{\partial \lambda}{\partial r}\right)\right], \\
G(l, l) & =R(n, n)=2 \frac{e^{-2 \lambda}}{r}\left(\frac{\partial v}{\partial r}+\frac{\partial \lambda}{\partial r}\right) \\
G(n, l) & =R\left(\zeta_{1}, \zeta_{1}\right)=R\left(\zeta_{2}, \zeta_{2}\right) \\
& =-\frac{e^{-2 \lambda}}{r}\left(\frac{\partial v}{\partial r}-\frac{\partial \lambda}{\partial r}\right)+\frac{1}{r^{2}}\left(1-e^{-2 \lambda}\right),
\end{aligned}
$$

and

$$
\begin{aligned}
G\left(\zeta_{1}, \zeta_{1}\right)= & G\left(\zeta_{2}, \zeta_{2}\right)=R(n, l)=-e^{-v-\lambda}\left(\frac{\partial^{2} v}{\partial u \partial r}+\frac{\partial^{2} \lambda}{\partial u \partial r}\right) \\
& +e^{-2 \lambda}\left[\frac{\partial^{2} v}{\partial r^{2}}+\left(\frac{\partial v}{\partial r}-\frac{\partial \lambda}{\partial r}\right)\left(\frac{1}{r}+\frac{\partial v}{\partial r}\right)\right] .
\end{aligned}
$$


The energy-momentum tensor of a massless scalar field is given by:

$$
T_{\mu \nu}=\partial_{\mu} \phi \partial_{v} \phi-\frac{1}{2} g_{\mu \nu} \sigma
$$

where

$$
\sigma=g^{\alpha \beta} \partial_{\alpha} \phi \partial_{\beta} \phi
$$

In the case considered here the scalar field is invariant under the rotation group. Thus, in the coordinate system of Sect. 1, $\phi$ is a function of $u$ and $r$ alone and

$$
\sigma=-2 e^{-v-\lambda} \frac{\partial \phi}{\partial u} \frac{\partial \phi}{\partial r}+e^{-2 \lambda}\left(\frac{\partial \phi}{\partial r}\right)^{2}
$$

In the null tetrad of Sect. 1, the only nonvanishing components of the energymomentum tensor are:

$$
\begin{aligned}
T(n, n) & =\left(e^{-v} \frac{\partial \phi}{\partial u}-\frac{1}{2} e^{-\lambda} \frac{\partial \phi}{\partial r}\right)^{2} \\
T(l, l) & =e^{-2 \lambda}\left(\frac{\partial \phi}{\partial r}\right)^{2}
\end{aligned}
$$

and

$$
T\left(\zeta_{1}, \zeta_{1}\right)=T\left(\zeta_{2}, \zeta_{2}\right)=-\frac{1}{2} \sigma
$$

Let $E_{\mu \nu}$ be the tensor,

$$
E_{\mu \nu}=G_{\mu \nu}-8 \pi T_{\mu \nu} .
$$

The Einstein equation are $E_{\mu \nu}=0$ or, equivalently, $R_{\mu \nu}=8 \pi \partial_{\mu} \phi \partial_{v} \phi$. In the present case the only nontrivial components of the tensor $E_{\mu \nu}$ are $E(n, n), E(n, l), E(l, l)$ and $E\left(\zeta_{1}, \zeta_{1}\right)=E\left(\zeta_{2}, \zeta_{2}\right)$

By virtue of the twice contacted Bianchi identities: $\nabla^{\mu} G_{\mu \nu} \equiv 0$, the integrability condition of the Einstein equations is the conservation of the energy-momentum tensor $\nabla^{\mu} T_{\mu \nu}=0$. For the energy-momentum tensor (2.11) we have:

$$
\nabla^{\mu} T_{\mu \nu}=\partial_{v} \phi \square_{g} \phi .
$$

Therefore, the integrability conditions is the wave equation for $\phi$ in the metric $g$, $\square_{g} \phi=0$, which in the case under consideration reads:

$$
-2\left(\frac{\partial^{2} \phi}{\partial u \partial r}+\frac{1 \partial \phi}{r} \frac{\partial u}{\partial u}+e^{v-\lambda}\left[\frac{\partial^{2} \phi}{\partial r^{2}}+\left(\frac{2}{r}+\frac{\partial v}{\partial r}-\frac{\partial \lambda}{\partial r}\right) \frac{\partial \phi}{\partial r}\right]=0 .\right.
$$

If the wave equation is satisfied, the twice contracted Bianchi identities become

$$
\nabla^{\mu} E_{\mu v}=0 \text {. }
$$

If, in addition, the Einstein equations $E(l, l)=0$ and $E(n, l)=0$ are satisfied, (2.20) yield $E\left(\zeta_{1}, \zeta_{1}\right)=E\left(\zeta_{2}, \zeta_{2}\right)=0$, and

$$
\frac{\partial E(n, n)}{\partial r}+2\left(\frac{\partial v}{\partial r}+\frac{1}{r}\right) E(n, n)=0 .
$$


From (2.21) we obtain

$$
E(n, n)(u, r)=-\frac{r_{0}^{2}}{r^{2}} \exp \left[2\left(v\left(u, r_{0}\right)-v(u, r)\right)\right] E(n, n)\left(u, r_{0}\right)
$$

If regularity at the center is assumed, (2.22) implies $E(n, n)=0$. We conclude that the Einstein equations $E(l, l)=0$ and $E(n, l)=0$, together with the wave equation, are equivalent to the full set of Einstein equations if regularity at the center is assumed.

\section{Section 3. The Nonlinear Evolution Equation}

The equation $E(l, l)=0$ reads

$$
\frac{\partial v}{\partial r}+\frac{\partial \lambda}{\partial r}=4 \pi r\left(\frac{\partial \phi}{\partial r}\right)^{2}
$$

This is an ordinary differential equation at each $u$. The solution which satisfies the asymptotic condition $v+\lambda \rightarrow 0$ as $r \rightarrow \infty$ is:

$$
v+\lambda=-4 \pi \int_{r}^{\infty} r\left(\frac{\partial \phi}{\partial r}\right)^{2} d r .
$$

The equation $E(n, l)=0$ reads:

$$
\frac{\partial v}{\partial r}-\frac{\partial \lambda}{\partial r}-\frac{1}{r}\left(e^{2 \lambda}-1\right)=0
$$

This is also an ordinary differential equation at each $u$. We can write it in the form

$$
\frac{\partial e^{v-\lambda}}{\partial r}=\frac{1}{r}\left(e^{v+\lambda}-e^{v-\lambda}\right)
$$

The only solution of this which exists for all $r \in[0, \infty[$ is

$$
e^{v-\lambda}=\frac{1}{r} \int_{0}^{r} e^{v+\lambda} d r
$$

Thus at each $u, e^{v+\lambda}$ is expressed in terms of $\phi$ by (3.2), and $e^{v-\lambda}$ is expressed in terms of $e^{v+\lambda}$ by (3.5). We can therefore substitute these expressions for the metric coefficients into the wave equation (2.19) obtaining a nonlinear partial integrodifferential equation for $\phi$. However, for reasons that will become evident in the sequel, we prefer to use as our principal unknown function the function $h$ defined by:

$$
h=\frac{\partial}{\partial r}(r \phi)
$$

We now introduce the following notation which will be used throughout: if $f$ is a function of $u$ and $r$, we denote by $\bar{f}$ the function of $u$ and $r$ which is at each $u$, the mean value of $f$ between 0 and $r$ :

$$
\bar{f}(u, r)=\frac{1}{r} \int_{0}^{r} f\left(u, r^{\prime}\right) d r^{\prime}
$$


We have

$$
f=\frac{\partial}{\partial r}(r \bar{f}) \text { or } \frac{\partial \bar{f}}{\partial r}=\frac{(f-\bar{f})}{r} .
$$

Thus in virtue of (3.6) we have:

$$
\phi=\bar{h} .
$$

Setting then

$$
e^{v+\lambda}=g,
$$

We have from (3.2):

and from (3.5)

$$
g=\exp \left[-4 \pi \int_{r}^{\infty}(h-\bar{h})^{2} \frac{d r}{r}\right]
$$

$$
e^{v-\lambda}=\bar{g} .
$$

We also define the differential operator

$$
D=\frac{\partial}{\partial u}-\frac{1}{2} \bar{g} \frac{\partial}{\partial r} .
$$

Since (see (1.2)) $D=e^{v} n$, the operator $D$ is the derivative along the incoming light rays parametrized by $u$. The wave equation (2.19) then becomes the nonlinear evolution equation

$$
D h=\frac{1}{2 r}(g-\bar{g})(h-\bar{h}) .
$$

By Sect. 2, this equation (with $g$ defined by (3.11)) has the whole content of the Einstein equations if regularity at the center is assumed: if $h$ is a solution of this equation, then $\phi, v$ and $\lambda$ defined by (3.9), (3.10) and (3.12) solve the Einstein equations. Conversely, if a spacetime satisfies the assumptions of Sect.1, and together with a scalar field $\phi$, is a solution of the Einstein equations, then $h$ defined by (3.6) solves the nonlinear evolution equation (3.13).

\section{Section 4. The Mass Equation}

The nonlinear evolution equation (3.13) gives the evolution law of the principal unknown function $h$ along the incoming light rays. From this equation we can derive the evolution law of any quantity which at each $u$ can be expressed in terms of the function $h$ at that $u$. We shall now derive the evolution law of $\bar{h}$. We have:

$$
\begin{aligned}
D(r \bar{h}) & =D\left(\int_{0}^{r} h d r\right)=\int_{0}^{r} \frac{\partial h}{\partial u} d r-\frac{1}{2} \bar{g} h=-\frac{1}{2} \bar{g} h+\int_{0}^{r}\left[\frac{1}{2} \frac{\partial \bar{g}}{\partial r}(h-\bar{h})+\frac{1}{2} \bar{g} \frac{\partial h}{\partial r}\right] d r \\
& =-\frac{1}{2}[\bar{g} h]_{r=0}-\frac{1}{2} \int_{0}^{r} \frac{\partial \bar{g}}{\partial r} \bar{h} d r \\
& =-\frac{1}{2} \bar{g} \bar{h}-\frac{1}{2}[\bar{g}(h-\bar{h})]_{r=0}+\frac{1}{2} \int_{0}^{r} \bar{g}(h-\bar{h}) \frac{d r}{r}
\end{aligned}
$$


and $D r=-\frac{1}{2} \bar{g}$. Now for any function $f$ which is continuous with respect to $r$ at $r=0$, we have $[f-\bar{f}]_{r=0}=0$. Thus, assuming regularity at the center, we conclude from the above that the evolution law of $h$ is:

$$
D h=\frac{\xi}{2 r},
$$

where

$$
\xi=\int_{0}^{r} \bar{g}(h-\bar{h}) \frac{d r}{r} .
$$

Let us now define the function $m$ by:

$$
m=\frac{r}{2}(1-\bar{g} / g)
$$

Since (see (3.11)) $g$ is a monotonically nondecreasing function of $r$ at each $u$, we have $\bar{g} \leqq g$. Therefore $m$ is nonnegative. Also, $m$ vanishes at $r=0$ and $m<r / 2$ for $r>0$. We have

$$
\frac{\partial m}{\partial r}=2 \pi \frac{\bar{g}}{g}(h-\bar{h})^{2} .
$$

Thus $m$ is a monotonically nondecreasing function of $r$ at each $u$, and we can write:

$$
m=2 \pi \int_{0}^{r} \frac{\bar{g}}{g}(h-\bar{h})^{2} d r
$$

The function $m$ an important physical meaning: $m(u, r)$ is the mass which at retarded time $u$ is contained within the sphere of radius $r$.

In terms of $m$ the Einstein equation $E(n, n)=0$ reads:

$$
D m=-\frac{4 \pi r^{2}}{g}(D \bar{h})^{2}
$$

which, by virtue of (4.1) is

$$
D m=-\frac{\pi}{g} \xi^{2}
$$

This is the evolution law of $m$, which according to our previous discussion, can be derived directly from the nonlinear evolution equation (3.13) if regularity at the center is assumed. It implies that $m$ is monotonically nonincreasing along the incoming light rays.

We shall assume that

$$
\lim _{r \rightarrow \infty} m(0, r):=M_{0}
$$

exists, that is that the initial total mass $M_{0}$ is finite. Then since $m(u, r)$ is monotonically nondecreasing with respect to $r$ at each $u$ and is bounded by $M_{0}$, the 
limit

$$
\lim _{r \rightarrow \infty} m(u, r):=M(u)
$$

exists for all $u \geqq 0 . M(u)$ is the total mass at retarded time $u$; this is the Bondi mass, first introduced in [4]. Equation (4.6) implies Bondi's theorem that $M$ is a monotonically nonincreasing function of $u, d M / d u \leqq 0$. In the present work the evolution law (4.6) of the local mass $m$ plays a very important role.

The finiteness of $M_{0}$ implies that at each $u, r(1-g) \rightarrow 0$ as $r \rightarrow \infty$. It then follows that we can express $M$ in the form:

$$
M=\frac{1}{2} \int_{0}^{\infty}(1-g) d r .
$$

Using now Eqs. (3.10), (3.11), (3.12), (3.13), (4.1), (4.2), (4.3) and (4.6), the nonvanishing components of the curvature tensor (see Sect. 2) are given by:

$$
\begin{gathered}
K\left(n, \zeta_{1}\right)=K\left(n, \zeta_{2}\right)=\frac{\pi \xi^{2}}{r^{2} g \bar{g}}, \\
K(n, l)=\frac{4 \pi}{r^{2} g}(h-\bar{h}) \xi-\frac{2 m}{r^{3}}, \\
K\left(l, \zeta_{1}\right)=K\left(l, \zeta_{2}\right)=\frac{4 \pi \bar{g}}{r^{2}}(h-\bar{g})^{2}, \\
K\left(\zeta_{1}, \zeta_{2}\right)=\frac{2 m}{r^{3}},
\end{gathered}
$$

and

$$
R\left(n, \zeta_{1}, l, \zeta_{1}\right)=R\left(n, \zeta_{2}, l, \zeta_{2}\right)=\frac{m}{r^{3}}
$$

The nonlinear evolution equation (3.13) is covariant under the scaling group $(u, r) \rightarrow(u / a, r / a)$, a being a positive real number. That is, if $h$ is a solution, then the function $h^{\prime}$ is defined by

$$
h^{\prime}(u, r)=h(u / a, r / a)
$$

is also a solution and we have:

$$
\bar{h}(u, r)=\bar{h}(u / a, r / a), \quad \bar{g}^{\prime}(u, r)=\bar{g}(u / a, r / a), \quad g^{\prime}(u, r)=g(u / a, r / a),
$$

and therefore

and

$$
m^{\prime}(u, r)=a m(u / a, r / a)
$$

$$
M^{\prime}(u)=a M(u / a)
$$

\section{Section 5. Properties of the Characteristics}

It is evident from the nonlinear evolution equation that the incoming light rays are the characteristics of our problem. They satisfy the ordinary differential equation

$$
\frac{d r}{d u}=-\frac{1}{2} \bar{g}(u, r)
$$


Let us denote by $\chi\left(u ; r_{0}\right)$ the characteristic through $r=r_{0}$ at $u=0$. From (5.1) we obtain:

$$
r_{1}:=\chi\left(u_{1} ; r_{0}\right)=r_{0}-\frac{1}{2} \int_{0}^{u_{1}} \bar{g}\left(u, \chi\left(u ; r_{0}\right)\right) d u .
$$

Since $\bar{g} \leqq g \leqq 1$ (see (3.11)), this implies

$$
r_{0} \leqq r_{1}+\frac{1}{2} u_{1} \text {. }
$$

Differentiating (5.2) with respect to $r_{0}$ we obtain

$$
\frac{\partial \chi\left(u_{1} ; r_{0}\right)}{\partial r_{0}}=1-\frac{1}{2} \int_{0}^{u_{1}} \frac{\partial \bar{g}}{\partial r}\left(u ; \chi\left(u ; r_{0}\right)\right) \frac{\partial \chi\left(u ; r_{0}\right)}{\partial r_{0}} d u .
$$

It follows that the function $\zeta(u, r)$ defined by

$$
\zeta\left(u_{1}, r_{1}\right)=\frac{\partial \chi\left(u_{1} ; r_{0}\right)}{\partial r_{0}}
$$

satisfies along the characteristics the evolution law

$$
D \zeta=-\frac{1}{2 r}(g-\bar{g}) \zeta
$$

and takes at $u=0$ the initial value $\zeta(0, r)=1$. Hence:

$$
\zeta\left(u_{1}, r_{1}\right)=\exp \left\{-\frac{1}{2} \int_{0}^{u_{1}}\left[\frac{1}{r}(g-\bar{g})\right]_{\chi\left(u ; r_{0}\right)} d u\right\} .
$$

We have $\zeta \leqq 1$ and also $\zeta\left(u_{1}, r_{1}\right)>0$, if $r_{1}>0$, since the integral can blow up only if $r_{1}=0$; in fact we have:

$$
\zeta\left(u_{1}, r_{1}\right) \geqq e^{-u_{1} / 2 r_{1}} .
$$

Consider now two characteristics $\chi\left(u ; r_{0}^{\prime}\right)$ and $\chi\left(u ; r_{0}\right)$ through $r=r_{0}^{\prime}$ and $r=r_{0}$ at $u=0$ respectively, where $r_{0}>r_{0}^{\prime}$. Then $r_{1} \geqq r_{1}^{\prime}$ and

$$
\frac{r_{1}-r_{1}^{\prime}}{r_{0}-r_{0}^{\prime}}=\frac{\chi\left(u_{1} ; r_{0}\right)-\chi\left(u_{1} ; r_{0}^{\prime}\right)}{r_{0}-r_{0}^{\prime}}=\underset{s \in\left[r_{0}^{\prime}, r_{0}\right]}{\operatorname{mean} \text { value }}\left\{\zeta\left(u_{1}, \chi\left(u_{1} ; s\right)\right)\right\} \leqq 1 .
$$

We conclude that the characteristics converge toward the future and the convergence factor is $\zeta$. The convergence factor plays a very important role in our work. The development of singularities in the domain of outer communications depends on the presence of points on the central line where $\zeta$ vanishes.

Let us return now to the mass equation (4.6). Integrating this equation along a characteristic, we obtain the mass-flux relation:

$$
m\left(u_{1}, r_{1}\right)+\pi \int_{0}^{u_{1}}\left[\frac{\xi^{2}}{g}\right]_{\chi} d u=m\left(0, r_{0}\right)
$$

The second term on the left represents physically the flux crossing the characteristic $\chi\left(u ; r_{0}\right)$ for $u$ between 0 and $u_{1}$. 


\section{Section 6. The Limiting Hypersurface $u=\infty$}

Since the Bondi mass $M(u)$ (see Sect. 4) is a nonegative monotonically nonincreasing function of $u$, the limit

$$
\lim _{u \rightarrow \infty} M(u):=M_{1}
$$

exists. $M_{1}$ is the final Bondi mass. We shall now show:

Proposition 1. For $r_{0}>2 M_{1}$ the timelike lines $r=r_{0}$ are complete toward the future.

Proof. The proper time element along the line $r=r_{0}$ is

$$
e^{v\left(u, r_{0}\right)} d u \text {. }
$$

From (3.10) and (3.12) we have $e^{2 v}=g \bar{g}$. Thus $v \leqq 0$ and

$$
e^{v} \geqq \bar{g} .
$$

Now since at each $u, \log \bar{g}(u, r) \rightarrow 0$ for $r \rightarrow \infty$, we can write (see (4.3))

$$
-\log \bar{g}\left(u, r_{0}^{\prime}\right)=\int_{r_{0}}^{\infty} \frac{1}{\bar{g}} \frac{\partial \bar{g}}{\partial r} d r=\int_{r_{0}}^{\infty} \frac{1}{\bar{g} r}(g-\bar{g}) d r=\int_{r_{0}}^{\infty} \frac{2 m}{r^{2}}\left(1-\frac{2 m}{r}\right)^{-1} d r .
$$

Thus, since $m(u, r) \leqq M(u)$, if $M(u)<r_{0} / 2$, we have

$$
-\log \bar{g}\left(u, r_{0}\right) \leqq \int_{r_{0}}^{\infty} \frac{2 M(u)}{r}\left(1-\frac{2 M(u)}{r}\right)^{-1} d r=-\log \left(1-\frac{2 M(u)}{r_{0}}\right) .
$$

Hence

$$
e^{v\left(u, r_{0}\right)} \geqq \bar{g}\left(u, r_{0}\right) \geqq 1-\frac{2 M(u)}{r_{0}} .
$$

Given now $r_{0}>2 M_{1}$, since $M(u) \rightarrow M_{1}$ for $u \rightarrow \infty$, there exists a $u_{2}$ such that for all $u \geqq u_{2}$, we have $M(u) \leqq \frac{1}{2}\left(r_{0} / 2+M_{1}\right)$. Thus for all $u \geqq u_{2}$ it holds:

$$
1-\frac{2 M(u)}{r_{0}} \geqq \frac{1}{2}\left(1-\frac{2 M_{1}}{r_{0}}\right) \text {. }
$$

Let then $u_{1}>u_{2}$. From (6.3) and the above we obtain:

$$
\begin{aligned}
& \int_{0}^{u_{1}} e^{v\left(u, r_{0}\right)} d u \geqq \int_{u_{2}}^{u_{1}} e^{v\left(u, r_{0}\right)} d u \geqq \int_{u_{2}}^{u_{1}}\left(1-\frac{2 M(u)}{r_{0}}\right) d u \\
& \geqq \frac{1}{2}\left(1-\frac{2 M_{1}}{r_{0}}\right)\left(u_{1}-u_{2}\right) \rightarrow \infty \text { for } u_{1} \rightarrow \infty .
\end{aligned}
$$

Consider now the limiting hypersurface $u=\infty$. By the above proposition the points of this hypersurface for which $r \geqq 2 M_{1}$ are at future timelike infinity $I^{+}$. As will be shown in a subsequent paper, if $M_{1} \neq 0$ the timelike lines $r=r_{0}$ for $r_{0}<2 M_{1}$ are incomplete, and the part of the limiting hypersurface $u=\infty$ for which $r<2 M_{1}$ represents the future event horizon $\mathrm{H}^{+}$. 


\section{Section 7. Local Existence of Classical Solutions}

In the following we shall study the initial value problem for the nonlinear evolution equation (3.13). Given initial data at $u=0$, that is a function $h(0, r) \in C^{1}[0, \infty$ [ and of finite initial Bondi mass $M_{0}$, we shall seek a solution to the equation for $u>0$, taking the given data at $u=0$. This means here that we are giving data on a future light cone with vertex at the center and we seek a solution in the interior of the cone. In this part of our work a "solution" will mean a "classical" solution, that is a differentiable function $h(u, r)$ whose derivatives are continuous even on the central line $r=0$, and which satisfies the equation.

For a classical solution of the nonlinear evolution equation the curvature tensor is continuous even at $r=0$, and the Einstein equations are satisfied in the classical sense. As we are interested in obtaining the fastest possible falloff toward null infinity, we shall assume that the initial data satisfies $h(0, r)=O\left(r^{-3}\right)$ and $\partial h / \partial r(0, r)$ $=O\left(r^{-4}\right)$. This is the fastest falloff class which is preserved by the evolution. The results can easily be generalized to the case when we only assume the much weaker condition that $M_{0}$ be finite. The aim of this paragraph is the proof of:

Theorem 1. For every initial data $h(0, r) \in C^{1}\left[0, \infty\left[\right.\right.$ such that $h(0, r)=0\left(r^{-3}\right)$ and $\partial h / \partial r(0, r)=O\left(r^{-4}\right)$ as $r \rightarrow \infty$, there exists a $u_{0}>0$ and a classical solution $h(u, r) \in C^{1}$ $\left[0, u_{0}\right] \times[0, \infty[$ of the nonlinear evolution equation, taking at $u=0$ the given data and such that at each $u \in\left[0, u_{0}\right], h(u, r)=O\left(r^{-3}\right)$ and $\partial h / \partial r(u, r)=O\left(r^{-4}\right)$ as $r \rightarrow \infty$, uniformly in $u$.

To prove this theorem we establish three lemmas. Let $X$ denote the space of functions $f$ defined on $\left[0, u_{0}\right] \times[0, \infty[$, which are continuous and posess a continuous partial derivative with respect to $r$ and for which

$$
\|f\|_{X}:=\sup _{u \in\left[0, u_{0}\right]} \sup _{r \geqq 0}\left\{(1+r)^{3}|f(u, r)|+(1+r)^{4}\left|\frac{\partial f}{\partial r}(u, r)\right|\right\}
$$

is finite. $X$ is a Banach space normed by \|\|$_{X}$. The proof of Theorem 1 uses an iteration method. Given $h_{n} \in X$ we define $h_{n+1}$ to be the solution of linear equation

$$
D_{n} h_{n+1}-\frac{1}{2 r}\left(g_{n}-\bar{g}_{n}\right) h_{n+1}=-\frac{1}{2 r}\left(g_{n}-\bar{g}_{n}\right) \bar{h}_{n},
$$

taking the given data at $u=0$. Here $g_{n}$ is the $g$-function corresponding to $h_{n}$ and $D_{n}$ is the $D$-operator corresponding to $g_{n}$. Starting from a $0^{\text {th }}$ iterate $h_{0} \in X$, we shall generate in this way a sequence $\left\{h_{n}\right\}$. We choose the $0^{t h}$ iterate to be:

$$
h_{0}(u, r)=h(0, r) \text {. }
$$

Then $\left\|h_{0}\right\|_{X}=d$, where

$$
d:=\sup _{r \geq 0}\left\{(1+r)^{3}|h(0, r)|+(1+r)^{4}\left|\frac{\partial h}{\partial r}(0, r)\right|\right\} .
$$

Lemma 1. For any $x_{1}>2 d$ there exists an $\eta>0, \eta=\eta\left(x_{1} ; d\right)$, such that if $u_{0}<\eta$ the sequence $\left\{h_{n}\right\}$ is contained in the closed ball of radius $x_{1}$ in the space $X$. 
Let now $Y$ denote the space of functions $f \in C^{0}\left[0, u_{0}\right] \times[0, \infty[$ such that

$$
\|F\|_{X}: \sup _{u \in\left[0, u_{0}\right]} \sup _{r \geqq 0}\left\{(1+r)^{3}|f(u, r)|\right\}
$$

is finite. $Y$ is a Banach space normed by \|\|$_{Y}$, and we have $Y \supset X$. In the following lemma it is assumed that $x_{1}$ and $u_{0}$ satisfy the inequalities of Lemma 1 .

Lemma 2. There exists a $\delta>0, \delta=\delta\left(x_{1}\right)$, such that if $u_{0}<\delta$ the sequence $\left\{h_{n}\right\}$ contracts with respect to the space $Y$.

From (7.2) it follows that the sequence $\left\{\partial h_{n} / \partial u\right\}$ is equibounded:

$$
\left|\frac{\partial h_{n+1}}{\partial u}\right| \leqq \frac{1}{2} x_{1}\left(1+\frac{\pi}{3} x_{1}^{2}\right) \cdot \frac{1}{(1+r)^{3}},
$$

in $\left[0, u_{0}\right] \times[0, \infty[$.

Lemma 3. The sequence $\left\{\partial h_{n} / \partial r\right\}$ is equicontinuous. Sketch of proof. Let $0 \leqq r_{1}^{\prime}<r_{1}$ and let $\chi_{n}\left(u ; r_{1}\right)$ and $\chi_{n}\left(n ; r_{1}^{\prime}\right)$ denote the characteristics $\chi_{n}$ through $r=r_{1}$ and $r=r_{1}^{\prime}$ respectively at $u=u_{1}$. We define

$$
\psi(u):=\frac{\partial h_{n+1}}{\partial r}\left(u, \chi_{n}\left(u ; r_{1}\right)\right)-\frac{\partial h_{n+1}}{\partial r}\left(u, \chi_{n}\left(u ; r_{1}^{\prime}\right)\right)
$$

We derive an ordinary differential inequality for $\psi$, which allows us to estimate the modulus of continuity with respect to $r$ of $\partial h_{n+1} / \partial r$ at $u=u_{1}$ in terms of the modulus of continuity of the data $\partial h(0, r) / \partial r$. This establishes the equicontinuity of the sequence $\left\{\partial h_{n} / \partial r\right\}$ with respect to $r$. The equicontinuity with respect to $u$ then follows, as, in view of Lemma 1 , the sequence $\left\{D_{n}\left(\partial h_{n+1} / \partial r\right)\right\}$ is equibounded.

Proof of Theorem 1. If $u_{0}<\min \{\eta, \delta\}$, the sequence $\left\{h_{n}\right\}$ is contained in the closed ball of radius $x_{1}$ in the space $X$ and contracts with respect to $Y$. Also, the family $\left\{h_{n}\right\}$ is equicontinuous and the family $\left\{\partial h_{n} / \partial r\right\}$ is equicontinuous as well. By the AscoliArzela theorem we can select a subsequence $\left\{h_{n_{i}}\right\}$ such that $h_{n_{i}} \rightarrow \tilde{h}$ and $\partial h_{n_{1}} / \partial r \rightarrow$ $\partial \tilde{h} / \partial r$ uniformly in compact subsets $\left[0, u_{0}\right] \times\left[0, r_{0}\right], r_{0}$ arbitrary; $\tilde{h}$ and $\partial \tilde{h} / \partial r$ are continuous functions in $\left[0, u_{0}\right] \times[0, \infty[$. It follows that

and

$$
\sup _{r \geqq 0}(1+r)^{3}|\tilde{h}(u, r)| \leqq x_{1}
$$

$$
\sup _{r \geqq 0}(1+r)^{4}\left|\frac{\partial \tilde{h}}{\partial r}(u, r)\right| \leqq x_{1} .
$$

Hence $\tilde{h} \in X$ and the convergence of $h_{n_{i}}$ and $\partial h_{n_{i}} / \partial r$ to $\tilde{h}$ and $\partial \tilde{h} / \partial r$ respectively, is uniform in $\left[0, u_{0}\right] \times\left[0, \infty\left[\right.\right.$. On the other hand, the contraction principle gives $h_{n} \rightarrow h$ in the space $Y$. Therefore $h=\bar{h} \in X$. We deduce further that $\bar{h}_{n_{i}}, g_{n_{i}}, \bar{g}_{n_{i}}$ and their derivatives with respect to $r$ converge uniformly to $\bar{h}, g, \bar{g}$, and their derivatives with respect to $r$.

We shall now show that the characteristics $\chi_{n_{i}}$ converge uniformly to the characteristics $\chi$. The characteristics $\chi_{n_{1}}\left(u ; r_{1}\right)$ and $\chi\left(u ; r_{1}\right)$ through $r=r_{1}$ at $u=u_{1}$ 
satisfy the ordinary differential equations

$$
\frac{d \chi_{n_{1}}}{d u}=-\frac{1}{2} \bar{g}_{n_{1}} \text { and } \quad \frac{d \chi}{d u}=-\frac{1}{2} \bar{g}
$$

respectively, together with the condition:

$$
\chi_{n_{i}}\left(u_{1} ; r_{1}\right)=\chi\left(u_{1} ; r_{1}\right)=r_{1} .
$$

We have

$$
\frac{d\left(\chi_{n_{i}}-\chi\right)}{d u}=-\frac{1}{2}\left[\bar{g}_{n_{i}}\left(u, \chi_{n_{i}}\right)-\bar{g}(u, \chi)\right],
$$

and $\left(\chi_{n_{i}}-\chi\right)\left(u_{1}\right)=0$. Thus, integrating, we obtain:

$$
\begin{aligned}
\left(\chi_{n_{i}}-\chi\right)(u)= & \frac{1}{2} \int_{u}^{u_{1}}\left[\bar{g}_{n_{i}}\left(u^{\prime}, \chi_{n_{i}}\right)-\bar{g}\left(u^{\prime}, \chi\right)\right] d u^{\prime} \\
= & \frac{1}{2} \int_{u}^{u_{1}}\left[\bar{g}_{n_{i}}\left(u^{\prime}, \chi\left(u^{\prime}\right)\right)-\bar{g}\left(u^{\prime}, \chi\left(u^{\prime}\right)\right)\right] d u^{\prime} \\
& +\frac{1}{2} \int_{u}^{u_{1}}\left[\bar{g}_{n_{i}}\left(u^{\prime}, \chi_{n_{i}}\left(u^{\prime}\right)\right)-\bar{g}_{n_{i}}\left(u^{\prime}, \chi\left(u^{\prime}\right)\right)\right] d u^{\prime} .
\end{aligned}
$$

We set

$$
y_{n_{i}}(u):=\left|\chi_{n_{i}}(u)-\chi(u)\right| .
$$

Then $y_{n_{i}}\left(u_{1}\right)=0$. By the uniform convergence of $\bar{g}_{n_{i}}$ to $\bar{g}$ we have $\mid \bar{g}_{n_{i}}\left(u^{\prime}, \chi\left(u^{\prime}\right)\right)$ $\bar{g}\left(u^{\prime}, \chi\left(u^{\prime}\right)\right) \mid \leqq \varepsilon_{n_{i}}$, where $\varepsilon_{n_{i}} \rightarrow 0$ for $i \rightarrow \infty$. Also by the equiboundedness of $\partial \bar{g}_{n_{i}} / \partial r$ we have:

$$
\left|\bar{g}_{n_{i}}\left(u^{\prime}, \chi_{n_{i}}\left(u^{\prime}\right)\right)-\bar{g}_{n_{i}}\left(u^{\prime}, \chi\left(u^{\prime}\right)\right)\right| \leqq a y_{n_{i}}\left(u^{\prime}\right) .
$$

Hence we obtain from (7.76) the inequality:

$$
y_{n_{i}}(u) \leqq \frac{1}{2} \varepsilon_{n_{i}}\left(u_{1}-u\right)+\frac{1}{2} a \int_{u_{1}}^{u} y_{n_{i}}\left(u^{\prime}\right) d u^{\prime}
$$

It follows that:

$$
y_{n_{1}}(u) \leqq \varepsilon_{n_{i}} \cdot \frac{1}{a}\left(e^{(1 / 2) a\left(u_{1}-u\right)}-1\right) .
$$

We conclude that $y_{n_{i}} \rightarrow 0$ uniformly in $u$ for $i \rightarrow \infty$ and therefore the characteristics $\chi_{n_{i}}$ converge uniformly to the characteristics $\chi$.

We have:

$$
\begin{aligned}
h_{n_{i}+1}\left(u_{1}, r_{1}\right)= & h\left(0, \chi_{n_{i}}\left(0 ; r_{1}\right)\right) e^{(1 / 2) \int_{0}^{u_{1}}\left[\left(\partial \bar{g}_{n_{i}} / \partial r\right)\right]_{\chi n_{i}} d u} \\
& +\int_{0}^{u_{1}} e^{(1 / 2) \int_{u}^{u_{1}}\left[\left(\partial \bar{g}_{\left.\left.n_{i} / \partial r\right)\right]_{n_{i}}} d u^{\prime}\right.\right.}\left[f_{n_{i}}\right]_{\chi_{n_{i}}} d u .
\end{aligned}
$$


Since $h_{n} \rightarrow h$ in the space $Y$, the left-hand side converges to $h\left(u_{1}, r_{1}\right)$ for $i \rightarrow \infty$, uniformly in $\left(u_{1}, r_{1}\right) \in\left[0, u_{0}\right][0, \infty]$. We now know also that for $i \rightarrow \infty$ the right-hand side converges to:

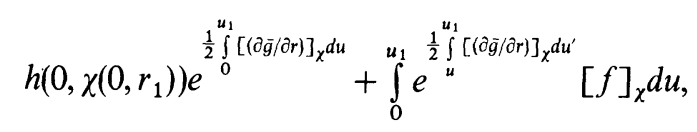

where

$$
f:=-\frac{1}{2} \frac{\partial \bar{g}}{\partial r} \bar{h}
$$

We conclude that the nonlinear evolution equation is satisfied in the integral sense in $\left[0, u_{0}\right] \times[0, \infty[$. In view of the fact that $\partial h / \partial r$ is known to be continuous, it follows that $h$ is continuously differentiable with respect to $u$ and satisfies the nonlinear evolution equation in the differential sense.

From this point, it can easily be shown that, for any $k \geqq 1$, if the initial data is of class $C^{k}$, then the above solution belongs to $C^{k}\left(\left[0, u_{0}\right] \times[0, \infty[)\right.$.

\section{Section 8. Global Uniqueness of Classical Solutions}

Theorem 2. Let $h_{1}$ and $h_{2}$ be two classical solutions of the nonlinear evolution equation in a strip $0 \leqq u \leqq u_{0}, u_{0}$ arbitrary, whose initial data coincide, $h_{1}(0, r)=h_{2}(0, r)$. Then, assuming that at each $u h_{1}, h_{2}=O\left(r^{-3}\right)$ and $\partial h_{1} / \partial r, \partial h_{2} / \partial r=O\left(r^{-4}\right)$ uniformly in $u$, we have $h_{1}=h_{2}$.

The proof is by deriving a linear homogeneous integral inequality for the quantity

$$
\Delta(u):=\sup _{r \geqq 0}\left\{(1+r)^{3}\left|\left(h_{1}-h_{2}\right)(u, r)\right|\right\} .
$$

\section{Section 9. Global Existence of Classical Solutions for Small Initial Data}

Theorem 3. We consider initial data $h(0, r) \in C^{1}\left[0, \infty\left[\right.\right.$ such that $h(0, r)=O\left(r^{-3}\right)$ and $\partial h / \partial r(0, r)=O\left(r^{-4}\right)$. Let $d_{0}$ denote

$$
d_{0}:=\inf _{a>0} \sup \left\{\left(1+\frac{r}{a}\right)^{3}|h(0, r)|+\left(1+\frac{r}{a}\right)^{4}\left|a \frac{\partial h}{\partial r}(0, r)\right|\right\} .
$$

Then, there exists a $\delta>0$ such that if $d_{0}<\delta$, there exists a global classical solution $h(u, r) \in C^{1}[0, \infty[\times[0, \infty[$ of the nonlinear evolution equation taking at $u=0$ the given data. This solution has the decay property:

$$
|h(u, r)| \leqq C(1+u+r)^{-3}, \quad|\partial h / \partial r(u, r)| \leqq C(1+u+r)^{-4} .
$$

The corresponding spacetime is timelike and null geodesically complete toward the future and

$$
M_{1}:=\lim _{u \rightarrow \infty} M(u)=0
$$


The quantity $d_{0}$ is a norm for the initial data which is invariant under the scaling group (see Sect. 4). The proof of Theorem 3 is based on two lemmas. Let $X^{\prime}$ denote the space of functions $f$ defined on $[0, \infty[\times[0, \infty[$, which are continuous and possess a continuous partial derivative with respect to $r$ and for which

$$
\|f\|_{X^{\prime}}:=\sup _{u \geqq 0} \sup _{r \geqq 0}\left\{\left(1+r+\frac{u}{2}\right)^{3}|h(u, r)|+\left(1+r+\frac{u}{2}\right)^{4}\left|\frac{\partial h}{\partial r}(u, r)\right|\right\}
$$

is finite. $X^{\prime}$ is a Banach space, normed by \|\|$_{X^{\prime}}$. The proof of Theorem 3 uses the same iteration method as that of Theorem 1 . Here however we choose the $0^{\text {th }}$ iterate to be:

$$
h_{0}(u, r)=h\left(0, r+\frac{u}{2}\right)
$$

Then we have $h_{0} \in X^{\prime}$ and $\left\|h_{0}\right\|_{X^{\prime}}=d$, where $d$ is defined by (7.4). Let $G(x), x \geqq 0$, be the function:

$$
G(x):=\frac{x}{\left(2+\frac{\pi}{6} x^{2}\right)}\left[e^{-(4 \pi / 3) x^{2}}-\frac{\pi}{8} x^{2}\left(2+\frac{\pi}{6} x^{2}\right)\right] .
$$

The function $G$ vanishes at $x=0$ and attains a maximum value $G=G_{0}>0$ at $x=x_{0}>0$. It is strictly monotonically increasing in the interval $\left[0, x_{0}\right]$.

Lemma 4. For each $\left.x \in] 0, x_{0}\right]$ the sequence $\left\{h_{n}\right\}$ is contained in the closed ball of radius $x$ in the space $X^{\prime}$ if $d \leqq G(x)$.

Proof. Let $\left\|h_{n}\right\|_{X^{\prime}} \leqq x$. We shall first derive global pointwise estimates for $h_{n}(u, r)$, $\left(h_{n}-\bar{h}_{n}\right)(u, r),\left(g_{n}-\bar{g}_{n}\right)(u, r)$, and a lower bound for $g_{n}(u, 0)$ for each $u \geqq 0$. We have

$$
\begin{aligned}
\left|h_{n}(u, r)\right| & \leqq \frac{1}{r} \int_{0}^{r}\left|h_{n}(u, s)\right| d s \leqq \frac{1}{r} \int_{0}^{r} \frac{\left\|h_{n}\right\|_{X^{\prime}}}{\left(1+\frac{u}{2} s\right)^{2}} d s \\
& \leqq \frac{x}{r} \cdot \frac{1}{2}\left[\frac{1}{\left(1+\frac{u}{2}\right)^{2}}-\frac{1}{\left(1+\frac{u}{2}+r\right)^{2}}\right] \\
& \leqq \frac{x}{\left(1+\frac{u}{2}\right)^{2}\left(1+\frac{u}{2}+r\right)}
\end{aligned}
$$

Also

$$
\begin{aligned}
\left|h_{n}(u, r)-h_{n}\left(u, r^{\prime}\right)\right| & \leqq \int_{r^{\prime}}^{r}\left|\frac{\partial h_{n}}{\partial s}(u, s)\right| d s \leqq\left\|h_{n}\right\|_{X^{\prime}} \int_{r^{\prime}}^{r} \frac{d s}{\left(1+s+\frac{u}{2}\right)^{4}} \\
& \leqq \frac{x}{3}\left[\frac{1}{\left(1+r^{\prime}+\frac{u}{2}\right)^{3}}-\frac{1}{\left(1+r+\frac{u}{2}\right)^{3}}\right]
\end{aligned}
$$


and therefore:

$$
\begin{aligned}
\left|\left(h_{n}-\bar{h}_{n}\right)(u, r)\right| & \leqq \frac{1}{r} \int_{0}^{r}\left|h_{n}(u, r)-h_{n}\left(u, r^{\prime}\right)\right| d r^{\prime} \\
& \leqq \frac{x}{3 r} \int_{0}^{r}\left[\frac{1}{\left(1+r^{\prime}+\frac{u}{2}\right)^{3}}-\frac{1}{\left(1+r+\frac{u}{2}\right)^{3}}\right] d r^{\prime} \\
& \leqq \frac{1}{2\left(1+\frac{u}{2}\right)^{2}\left(1+\frac{u}{2}+r\right)^{2}} \cdot x
\end{aligned}
$$

We then have

$$
\int_{0}^{\infty}\left(h_{n}-\bar{h}_{n}\right)^{2} \frac{d r}{r} \leqq \frac{x^{2}}{4} \int_{0}^{\infty} \frac{r d r}{\left(1+\frac{u}{2}\right)^{4}\left(1+\frac{u}{2}+r\right)^{4}}=\frac{1}{24} \frac{1}{\left(1+\frac{u}{2}\right)^{6}} \cdot x^{2}
$$

Hence

$$
g_{n}(u, 0) \geqq \exp \left(-\frac{\pi}{6} \frac{x}{\left(1+\frac{u}{2}\right)^{6}}\right)
$$

Taking into account (9.6) we find:

and therefore

$$
\begin{aligned}
\int_{r^{\prime}}^{r} \frac{\partial g_{n}}{\partial s}(u, s) d s \leqq & 4 \pi \int_{r^{\prime}}^{r}\left(h_{n}-h_{n}\right)^{2} \frac{d s}{s} \leqq \pi x^{2} \int_{r^{\prime}}^{r} \frac{s d s}{\left(1+\frac{u}{2}\right)^{4}\left(1+\frac{u}{2}+s\right)^{4}} \\
= & \frac{\pi x^{2}}{\left(1+\frac{u}{2}\right)^{4}}\left\{\frac{1}{2}\left[\frac{1}{\left(1+\frac{u}{2}+r^{\prime}\right)^{2}}-\frac{1}{\left(1+\frac{u}{2}+r\right)^{2}}\right]\right. \\
& \left.-\frac{\left(1+\frac{u}{2}\right)}{3}\left[\frac{1}{\left(1+\frac{u}{2}+r^{\prime}\right)^{3}}-\frac{1}{\left(1+\frac{u}{2}+r\right)^{3}}\right]\right\},
\end{aligned}
$$

$$
\begin{aligned}
\left(g_{n}-\bar{g}_{n}\right)(u, r)= & \frac{1}{r} \int_{0}^{r}\left\{\int_{r^{\prime}}^{r} \frac{\partial g_{n}}{\partial s}(u, s) d s\right\} d r^{\prime} \\
\leqq & \frac{\pi x^{2}}{\left(1+\frac{u}{2}\right)^{4}} \cdot \frac{1}{4}\left\{\frac{1}{2} \int_{0}^{r}\left[\frac{1}{\left(1+\frac{u}{2}+r^{\prime}\right)^{2}}-\frac{1}{\left(1+\frac{u}{2}+r\right)^{2}}\right] d r^{\prime}\right. \\
& \left.-\frac{1}{3}\left(1+\frac{u}{2}\right) \int_{0}^{r}\left[\frac{1}{\left(1+\frac{u}{2}+r^{\prime}\right)^{3}}-\frac{1}{\left(1+\frac{u}{2}+r\right)^{3}}\right] d r^{\prime}\right\}
\end{aligned}
$$




$$
=\frac{\pi}{3} x^{2} \frac{\pi}{\left(1+\frac{u}{2}\right)^{5}\left(1+\frac{u}{2}+r\right)^{3}} .
$$

Let $f_{n}$ denote the right-hand side of (7.2), $f_{n}:=-1 / 2 r\left(g_{n}-\bar{g}_{n}\right) \bar{h}_{n}$. From (9.9) and (9.4) we obtain:

$$
\left|f_{n}(u, r)\right| \leqq \frac{\pi}{6} x^{3} \cdot \frac{r}{\left(1+\frac{u}{2}\right)^{7}\left(1+\frac{u}{2}+r\right)^{4}}
$$

and we can estimate $f_{n}$ by a monotonically decreasing function of $r$ at each $u$ :

$$
\left|f_{n}(u, r)\right| \leqq \frac{\pi}{6} x^{3} \cdot \frac{1}{\left(1+\frac{u}{2}\right)^{7}\left(1+\frac{u}{2}+r\right)^{3}}
$$

Since by (9.7) it holds:

$$
\bar{g}_{n}(u, r) \geqq g_{n}(u, 0) \geqq \exp \left(-\frac{\pi}{6} \frac{x^{2}}{\left.\left(1+\frac{u}{2}\right)^{6}\right)},\right.
$$

we conclude that the characteristics $\chi_{n}$ of the linear equation (7.2) obey the inequality

$$
r(u)=r_{1}+\frac{1}{2} \int_{u}^{u_{1}} \bar{g}_{n}\left(u^{\prime}, r\left(u^{\prime}\right)\right) d u^{\prime} \geqq r_{1}+\frac{1}{2} k\left(u_{1}-u\right),
$$

where $k:=e^{-(\pi / 6) x^{2}}$. We shall now estimate the integral of $f_{n}$ along the characteristics $\chi_{n}$. From (9.10) we have:

$$
\begin{aligned}
\int_{0}^{u_{1}}\left[\left|f_{n}\right|\right]_{x_{n}} d u & \leqq \frac{\pi}{6} x^{3} \int_{0}^{u_{1}} \frac{d u}{\left(1+\frac{u}{2}\right)^{7}\left(1+\frac{u}{2}+r(u)\right)^{3}} \\
& \leqq \frac{\pi}{6} x^{3} \int_{0}^{u_{1}} \frac{d u}{\left(1+\frac{u}{2}\right)^{7}\left(1+\frac{u}{2}+r_{1}+\frac{k}{2}\left(u_{1}-u\right)\right)^{3}}
\end{aligned}
$$

Now since $k \leqq 1$, it holds:

$$
1+\frac{u}{2}+r_{1}+\frac{k}{2}\left(u_{1}-u\right) \geqq k\left(1+\frac{u_{1}}{2}+r_{1}\right) .
$$

Thus, using the fact that

$$
\int_{0}^{\infty} \frac{d u}{\left(1+\frac{u}{2}\right)^{7}}=\frac{1}{3}
$$


we obtain:

$$
\int_{0}^{u_{1}}\left[\left|f_{n}\right|_{x_{n}}\right] d u \leqq \frac{\pi x^{3}}{6} \frac{1}{k^{3}} \frac{1}{\left(1+\frac{u_{1}}{2}+r_{1}\right)^{3}} \int_{0}^{u_{1}} \frac{d u}{\left(1+\frac{u}{2}\right)^{7}} \leqq \frac{\pi}{18} \frac{x^{3}}{k^{3}} \frac{1}{\left(1+\frac{u_{1}}{2}+r_{1}\right)^{3}} .
$$

Since by (9.9) it holds:

$$
\frac{1}{r}\left(g_{n}-\bar{g}_{n}\right) \leqq \frac{\pi}{3} x^{2} \cdot \frac{1}{\left(1+\frac{u}{2}\right)^{7}}
$$

we have:

$$
\int_{0}^{u_{1}}\left[\frac{1}{r}\left(g_{n}-\bar{g}_{n}\right)\right]_{x_{n}} d u \leqq \frac{\pi}{3} x^{2} \int_{0}^{u_{1}} \frac{d u}{\left(1+\frac{u}{2}\right)^{7}} \leqq \frac{\pi}{9} x^{2} .
$$

Also, taking into account the fact that $r_{0} \geqq r_{1}+\frac{1}{2} k u_{1}$ and $k \leqq 1$, we obtain:

$$
\left|h\left(0, r_{0}\right)\right| \leqq \frac{d}{\left(1+r_{0}\right)^{3}} \leqq \frac{d}{\left(1+r_{1}+\frac{1}{2} k u_{1}\right)^{3}} \leqq \frac{d}{k^{3}} \cdot \frac{1}{\left(1+\frac{u_{1}}{2}+r_{1}\right)^{3}} .
$$

We now integrate the linear equation (7.2) along the characteristics $\chi_{n}$ to obtain:

$$
h_{n+1}\left(u_{1}, r_{1}\right)=h\left(0, r_{0}\right) e^{\int_{0}^{u_{1}}\left[(1 / 2 r)\left(g_{n}-\bar{g}_{n}\right)\right]_{x n} d u}+\int_{0}^{u_{1}} e^{\int_{1}^{u}\left[(1 / 2 r)\left(g_{n}-\bar{g}_{n}\right)\right]_{x n} d u^{\prime}}\left[f_{n}\right]_{\chi_{n}} d u,
$$

where $r_{0}:=r(0)$. Using (9.13), (9.14) and (9.12), we then conclude that for all $u_{1}$, $r_{1} \geqq 0$, it holds:

$$
\left(1+r_{1}+\frac{u_{1}}{2}\right)^{3}\left|h_{n+1}\left(u_{1}, r_{1}\right)\right| \leqq e^{(5 \pi / 9) x^{2}}\left(d+\frac{\pi}{18} x^{3}\right)
$$

Differentiating now the linear equation (7.2) with respect to $r$ we obtain:

$$
D_{n}\left(\frac{\partial h_{n+1}}{\partial r}\right)-\frac{1}{r}\left(g_{n}-\bar{g}_{n}\right) \frac{\partial h_{n+1}}{\partial r}=f_{n^{\prime}}^{\prime},
$$

where

$$
f_{n}^{\prime}:=\frac{\partial f_{n}}{\partial r}+\frac{1}{2} \frac{\partial^{2} \bar{g}_{n}}{\partial r^{2}} h_{n+1}
$$

We have

$$
\frac{\partial f_{n}}{\partial r}=-\frac{1}{2} \frac{\partial^{2} \bar{g}_{n}}{\partial r^{2}} \bar{h}_{n}-\frac{1}{2} \frac{\partial \bar{g}_{n}}{\partial r} \frac{\left(h_{n}-\bar{h}_{n}\right)}{r}
$$


We have

$$
\frac{\partial^{2} \bar{g}_{n}}{\partial r^{2}}=-\frac{2}{r^{2}}\left(g_{n}-\bar{g}_{n}\right)+\frac{4 \pi}{r^{2}}\left(h_{n}-\bar{h}_{n}\right)^{2} g_{n}
$$

From (9.9) and (9.6) we then obtain:

$$
\left|\frac{\partial^{2} \bar{g}_{n}}{\partial r^{2}}\right| \leqq \pi x^{2} \cdot \frac{1}{\left(1+\frac{u}{2}\right)^{5}\left(1+\frac{u}{2}+r\right)^{3}}
$$

Taking this into account together with (9.4), (9.9) and (9.6), we obtain

$$
\left|\frac{\partial f_{n}}{\partial r}\right| \leqq \frac{7}{12} \pi x^{3} \cdot \frac{1}{\left(1+\frac{u}{2}\right)^{7}\left(1+\frac{u}{2}+r\right)^{4}}
$$

Also the estimate $(9.15)$ gives:

$$
\left|\frac{\partial^{2} \bar{g}_{n}}{\partial r^{2}}\right|\left|h_{n+1}\right| \leqq \pi x^{2}\left(d+\frac{\pi}{18} x^{3}\right) e^{(5 \pi / 9) x^{2}} \cdot \frac{1}{\left(1+\frac{u}{2}\right)^{5}\left(1+\frac{u}{2}+r\right)^{6}}
$$

From (9.17) and (9.18) we conclude that we can estimate $f_{n}^{\prime}$ by a monotonically decreasing function of $r$ at each $u$,

$$
\left|f_{n}^{\prime}\right| \leqq \frac{\Lambda^{\prime}}{\left(1+\frac{u}{2}\right)^{7}\left(1+\frac{u}{2}+r\right)^{4}}
$$

where

$$
\Lambda^{\prime}:=\frac{7}{12} \pi x^{3}+\frac{\pi}{2} x^{2}\left(d+\frac{\pi}{18} x^{3}\right) e^{(5 \pi / 9) x^{2}} .
$$

Using now (9.11) we can estimate the integral of $f_{n}^{\prime}$ along the characteristics $\chi_{n}$,

$$
\begin{aligned}
\int_{0}^{u_{1}}\left[\left(f_{n}^{\prime}\right)\right]_{x_{n}} d u & \leqq \Lambda^{\prime} \int_{0}^{u_{1}} \frac{d u}{\left(1+\frac{u}{2}\right)^{7}\left(1+\frac{u}{2}+r(u)\right)^{4}} \\
& \leqq \Lambda^{\prime} \cdot \frac{1}{k^{4}} \cdot \frac{1}{\left(1+\frac{u_{1}}{2}+r_{1}\right)^{4}} \int_{0}^{u_{1}} \frac{d u}{\left(1+\frac{u}{2}\right)^{7}} \leqq \frac{\Lambda^{\prime}}{3 k^{4}} \cdot \frac{1}{\left(1+\frac{u_{1}}{2}+r_{1}\right)^{4}}
\end{aligned}
$$

Also, as in (9.14) we have:

$$
\left|\frac{\partial h}{\partial r}\left(0, r_{0}\right)\right| \leqq \frac{d}{\left(1+r_{0}\right)^{4}} \leqq \frac{d}{k^{4}} \cdot \frac{1}{\left(1+\frac{u_{1}}{2}+r_{1}\right)^{4}}
$$


Integrating (9.16) along the characteristics $\chi_{n}$ we obtain:

$$
\begin{aligned}
\frac{\partial h_{n+1}}{\partial r}\left(u_{1}, r_{1}\right)= & \frac{\partial h}{\partial r}\left(0, r_{0}\right) e^{\int_{0}^{u_{1}}\left[(1 / r)\left(g_{n}-\bar{g}_{n}\right)\right]_{x_{n}} d u^{\prime}} \\
& +\int_{0}^{u_{1}} e^{\int_{1}^{u}\left[(1 / r)\left(g_{n}-\bar{g}_{n}\right)\right]_{\chi_{n}} d u^{\prime}}\left[f_{n}^{\prime}\right]_{\chi_{n}} d u .
\end{aligned}
$$

Using (9.13), (9.21) and (9.22), we then conclude that for all $u_{1}, r_{1} \geqq 0$ it holds:

$$
\begin{aligned}
& \left(1+r_{1}+\frac{u_{1}}{2}\right)^{4}\left|\frac{\partial h_{n+1}}{\partial r}\left(u_{1}, r_{1}\right)\right| \\
& \leqq e^{(7 \pi / 9) x^{2}}\left\{d+\frac{\pi}{6} x^{2}\left[\frac{7}{6} x+\left(d+\frac{\pi}{18} x^{3}\right) e^{(5 \pi / 9) x^{2}}\right]\right\} .
\end{aligned}
$$

Adding finally the estimate (9.15) and (9.23) we conclude:

$$
\left\|h_{n+1}\right\|_{X^{\prime}} \leqq e^{(5 \pi / 9) x^{2}}\left(d+\frac{\pi}{18} x^{3}\right)+e^{(7 \pi / 9) x^{2}}\left\{d+\frac{\pi}{6} x^{2}\left[\frac{7}{6} x+\left(d+\frac{\pi}{18} x^{3}\right) e^{(5 \pi / 9) x^{2}}\right]\right\} \text {. }
$$

We simplify to

$$
\left\|h_{n+1}\right\|_{X^{\prime}} \leqq\left(2+\frac{\pi}{6} x^{2}\right) e^{(4 \pi / 3) x^{2}}\left(d+\frac{\pi}{8} x^{3}\right) .
$$

Hence if $\|h\|_{X^{\prime}} \leqq x$, this inequality implies that also $\left\|h_{n+1}\right\| \leqq x$, provided that $d \leqq G(x)$, where $G$ is the function given by (9.3). Since then $x>2 d$, the $0^{\text {th }}$ iterate (9.2) is such that $\left\|h_{0}\right\|_{X^{\prime}} \leqq x$. We conclude by induction that the whole sequence of iterates is contained in the closed ball of radius $x$ in the space $X^{\prime}$.

Let now $Y^{\prime}$ denote the space of functions $f \in C^{0}[0, \infty[\times[0, \infty[$ such that

$$
\|f\|_{Y^{\prime}}:=\sup _{u \geqq 0} \sup _{r \geqq 0}\left\{\left(1+r+\frac{u}{2}\right)^{3}|f(u, r)|\right\}
$$

is finite. $Y^{\prime}$ is a Banach space normed by $\|\quad\|_{Y^{\prime}}$ and we have $Y^{\prime} \supset X^{\prime}$. Let $s(x), x \geqq 0$, be the function

$$
S(x):=\frac{1}{3} \pi x^{2}\left(\frac{37}{6}+2 \pi x^{2}\right) e^{(5 \pi / 9) x^{2}} .
$$

The function $s$ is strictly monotonically increasing and vanishes at $x=0$. Let $x$ be the solution of $s(x)=1$. Then $x_{1}$ is a positive real number. In the following lemma it is assumed that $x$ and $d$ satisfy the inequality of Lemma 4.

Lemma 5. If $x<x_{1}$, the sequence $\left\{h_{n}\right\}$ contracts with respect to the space $Y^{\prime}$.

Proof. We have

$$
\begin{aligned}
& D_{n}\left(h_{n+1}-h_{n}\right)-\frac{1}{2} \frac{\partial \bar{g}_{n}}{\partial r}\left(h_{n+1}-h_{n}\right)=\frac{1}{2}\left(\bar{g}_{n}-\bar{g}_{n-1}\right) \frac{\partial h_{n}}{\partial r} \\
& +\frac{1}{2} h_{n}\left(\frac{\partial \bar{g}_{n}}{\partial r}-\frac{\partial \bar{g}_{n-1}}{\partial r}\right)+f_{n}-f_{n-1},
\end{aligned}
$$


and

$$
f_{n}-f_{n-1}=-\frac{1}{2} \frac{\partial \bar{g}_{n}}{\partial r}\left(\bar{h}_{n}-\bar{h}_{n-1}\right)-\frac{1}{2} \bar{h}_{n-1}\left(\frac{\partial \bar{g}_{n}}{\partial r}-\frac{\partial \bar{g}_{n-1}}{\partial r}\right) .
$$

Now, from (9.6),

$$
\left|h_{n}-\bar{h}_{n}+h_{n-1}-\bar{h}_{n-1}\right| \leqq \frac{r}{\left(1+\frac{u}{2}\right)^{2}\left(1+\frac{u}{2}+r\right)^{2}} x,
$$

and we can estimate

$$
\left|h_{n}-h_{n-1}-h_{n}+h_{n+1}\right| \leqq 2 \frac{\left\|h_{n}-h_{n-1}\right\|_{Y^{\prime}}}{\left(1+\frac{u}{2}\right)\left(1+\frac{u}{2}+r\right)^{2}}
$$

Thus, multiplying,

$$
\left|\left(h_{n}-\bar{h}_{n}\right)^{2}-\left(h_{n-1}-\bar{h}_{n-1}\right)^{2}\right| \leqq \frac{2 r}{\left(1+\frac{u}{2}\right)^{4}\left(1+\frac{u}{2}+r\right)^{3}} x\left\|h_{n}-h_{n-1}\right\|_{Y^{\prime}}
$$

We then obtain

$$
\begin{aligned}
\left|A_{n}-A_{n-1}\right| & \leqq x\left\|h_{n}-h_{n-1}\right\|_{Y^{\prime}} \int_{r}^{\infty} \frac{2 d r}{\left(1+\frac{u}{2}\right)^{4}\left(1+\frac{u}{2}+r\right)^{3}} \\
& =x\left\|h_{n}-h_{n-1}\right\|_{Y^{\prime}} \cdot \frac{1}{\left(1+\frac{u}{2}\right)^{4}\left(1+\frac{u}{2}+r\right)^{2}}
\end{aligned}
$$

and therefore

$$
\left|g_{n}-g_{n-1}\right| \leqq 4 \pi x\left\|h_{n}-h_{n-1}\right\|_{Y^{\prime}} \cdot \frac{1}{\left(1+\frac{u}{2}\right)^{4}\left(1+\frac{u}{2}+r\right)^{2}}
$$

It follows that

$$
\left|\bar{g}_{n}-\bar{g}_{n-1}\right| \leqq \frac{1}{r} \int_{0}^{r}\left|g_{n}-g_{n-1}\right| d r \leqq 4 \pi x\left\|h_{n}-h_{n-1}\right\|_{Y^{\prime}} \frac{1}{\left(1+\frac{u}{2}\right)^{5}\left(1+\frac{u}{2}+r\right)}
$$

Using now (9.29), (9.30) and (9.6), we obtain:

$$
\frac{1}{4 \pi}\left|\frac{\partial\left(g_{n}-g_{n-1}\right)}{\partial r}\right| \leqq x\left(2+\pi x^{2}\right)\left\|h_{n}-h_{n-1}\right\|_{Y^{\prime}} \frac{1}{\left(1+\frac{u}{2}\right)^{4}\left(1+\frac{u}{2}+r\right)^{3}}
$$


Since

$$
\int_{0}^{r} \int_{r^{\prime}}^{r} \frac{d s}{\left(1+\frac{u}{2}+s\right)^{3}} d r^{\prime}=\frac{1}{2} \frac{r^{2}}{\left(1+\frac{u}{2}\right)\left(1+\frac{u}{2}+r\right)^{2}},
$$

we obtain from (9.32):

$$
\begin{aligned}
\left|\frac{\partial \bar{g}_{n}}{\partial r}-\frac{\partial \bar{g}_{n-1}}{\partial r}\right| & \leqq \frac{1}{r^{2}} \int_{0}^{r}\left\{\int_{r^{\prime}}^{r}\left|\frac{\partial\left(g_{n}-g_{n-1}\right)}{\partial s}\right| d s\right\} d r^{\prime} \\
& \leqq 2 \pi x\left(2+\pi x^{2}\right)\left\|h_{n}-h_{n-1}\right\|_{X^{\prime}} \frac{1}{\left(1+\frac{u}{2}\right)^{5}\left(1+\frac{u}{2}+r\right)^{2}}
\end{aligned}
$$

Taking into account this, we deduce from (9.28):

$$
\left|f_{n}-f_{n-1}\right| \leqq \pi x^{2}\left(\frac{13}{6}+\pi x^{2}\right)\left\|h_{n}-h_{n-1}\right\|_{Y^{\prime}} \frac{1}{\left(1+\frac{u}{2}\right)^{7}\left(1+\frac{u}{2}+r\right)^{3}}
$$

Also, by (9.33) and (9.31) we have

$$
\frac{1}{2}\left|\bar{g}_{n}-\bar{g}_{n-1}\right|\left|\frac{\partial h_{n}}{\partial r}\right| \leqq 2 \pi x^{2}\left\|h_{n}-h_{n-1}\right\|_{Y^{\prime}} \frac{1}{\left(1+\frac{u}{2}\right)^{5}\left(1+\frac{u}{2}+r\right)^{5}}
$$

and

$$
\frac{1}{2}\left|h_{n}\right|\left|\frac{\partial \bar{g}_{n}}{\partial r}-\frac{\partial \bar{g}_{n-1}}{\partial r}\right| \leqq \pi x^{2}\left(2+\pi x^{2}\right)\left\|h_{n}-h_{n-1}\right\|_{Y^{\prime}} \frac{1}{\left(1+\frac{u}{2}\right)^{5}\left(1+\frac{u}{2}+r\right)^{5}}
$$

Using now (9.35), (9.36) and (9.34), we obtain from (9.27):

$$
\begin{gathered}
\left|D_{n}\left(h_{n+1}-h_{n}\right)-\frac{1}{2} \frac{\partial \bar{g}_{n}}{\partial r}\left(h_{n+1}-h_{n}\right)\right| \leqq \\
\qquad x^{2}\left(\frac{37}{6}+2 \pi x^{2}\right)\left\|h_{n}-h_{n-1}\right\|_{Y^{\prime}} \\
\cdot \frac{1}{\left(1+\frac{u}{2}\right)^{7}\left(1+\frac{u}{2}+r\right)^{3}} .
\end{gathered}
$$

Integrating this inequality along the characteristics $\chi_{n}$ we obtain, in a way similar to that in the first part of the proof of Lemma 4 , that for all $u_{1}, r_{1} \geqq 0$, it holds:

$$
\begin{aligned}
\left.\left(1+r_{1}+\frac{u_{1}}{2}\right)^{3} \mid h_{n+1}-h_{n}\right)\left(u_{1}, r_{1}\right) \mid \leqq & e^{(\pi / 18) x^{2}} \cdot \frac{1}{k^{3}} \cdot \frac{1}{3} \pi x^{2} \\
& \cdot\left(\frac{37}{6}+2 \pi x^{2}\right)\left\|h_{n}-h_{n-1}\right\|_{Y^{\prime}}
\end{aligned}
$$


Since here $\left(h_{n+1}-h_{n}\right)(0, r)=0$, we conclude that

$$
\left\|h_{n+1}-h_{n}\right\|_{Y^{\prime}} \leqq s(x)\left\|h_{n}-h_{n-1}\right\|_{Y^{\prime}}
$$

where $s$ is the function given by (9.26). If $x<x_{1}$, then $s(x)<1$ and (9.38) implies that the sequence $\left\{h_{n}\right\}$ contracts with respect to the space $Y^{\prime}$.

Proof of Theorem 3. We define

$$
\delta:=\max _{x \in\left[0, x_{1}\right]}\{G(x)\}
$$

$\delta$ is a positive real number and if $d<\delta$, then we can find $x<x_{1}$ such that $d \leqq G(x)$. Then by Lemma 4 the sequence $\left\{h_{n}\right\}$ is contained in the closed ball of radius $x$ in the space $X^{\prime}$, and by Lemma 5 the sequence contracts with respect to the space $Y^{\prime}$. Under these conditions the argument of Lemma 3 will show that the sequence $\left\{\partial h_{n} / \partial r\right\}$ is equicontinuous. Then the argument in the proof of Theorem 1 will show that the limit $h$ of the sequence $\left\{h_{n}\right\}$ belongs to the space $X^{\prime}$ and is continuously differentiable with respect to $u$ and satisfies the nonlinear evolution equation in the differential sense. Thus $h$ is a global classical solution.

Let us now be given initial data $h(0, r)$ such that

$$
d_{0}<\delta \text {. }
$$

Then there exists an $a>0$ such that

$$
\sup _{r \geqq 0}\left\{\left(1+\frac{r}{a}\right)^{3}|h(0, r)|+\left(1+\frac{r}{a}\right)^{4}\left|a \frac{\partial h}{\partial r}(0, r)\right|\right\}<\delta .
$$

We define new initial data $h^{\prime}(0, r)$ by:

$$
h^{\prime}(0, r)=h(0, a r)
$$

Then

$$
\begin{aligned}
d^{\prime}: & =\sup _{r \geqq 0}\left\{(1+r)^{3}\left|h^{\prime}(0, r)\right|+(1+r)^{4}\left|\frac{\partial h^{\prime}}{\partial r}(0, r)\right|\right\} \\
& =\sup _{r \geqq 0}\left\{\left(1+\frac{r}{a}\right)^{3}|h(0, r)|+\left(1+\frac{r}{a}\right)^{4}\left|a \frac{\partial h}{\partial r}(0, r)\right|\right\} .
\end{aligned}
$$

Thus we have $d^{\prime}<\delta$. By the above this implies that we have a global classical solution $h^{\prime}(u, r)$ which belongs to the space $X^{\prime}$ and takes at $u=0$ the data $h^{\prime}(0 . r)$. Hence, by the scaling group covariance (see Sect.4), the function $h$ defined by $h(u, r)=h^{\prime}(u / a, r / a)$ is a global classical solution taking at $u=0$ the given data $h(0, r)$. This solution has the decay property:

$$
\left|h(u, r) \leqq C(1+u+r)^{-3},\right| \partial h(u, r) \mid \leqq C(1+u+r)^{-4} .
$$

The remaining conclusions of the theorem follow from the corresponding decay properties of the metric functions and their derivatives. 


\section{References}

1. Penrose, R.: Gravitational collapse and space-time singularities. Phys. Rev. Lett. 14, 57-59 (1965)

2. Hawking, S. W., Penrose, R.: The singularities of gravitational collapse and cosmology. Proc. Roy. Soc. Lond. A 314, 529-548 (1970)

3. Hawking, S. W., Ellis G. F. R.: The large scale structure of space-time. Cambridge: Cambridge University Press 1973

4. Bondi, H., van Burg, M. G. J., Metzner, A. W. K.: Gravitational waves in general relativity VII. Waves from axi-symmetric isolated systems. Proc. Roy. Soc. Lond. A269, 21-48 (1962)

Communicated by S.-T. Yau

Received November 9, 1984; in revised form December 18, 1985 
